\title{
STABILIZATION OF LINEAR SYSTEMS
}

\author{
Vanita Jain \\ Bharati Vidyapeeth College of Engineering, New Delhi
}

\begin{abstract}
Simultaneous stabilization is recognized as one of the open issues in linear system theory, arises for instance in many applications such as reliable control, robust control and nonlinear control. This paper deals with designing a constant output feedback controller for the stabilization of given $\mathrm{M}$ linear systems. The design equations are functions of the state and control weighting matrices.
\end{abstract}

Keywords- controller, linear, time-invariant, simultaneous stabilization

\section{I.INTRODUCTION}

The problem of simultaneously stabilizing a family of linear continuous systems by an output controller is considered here. The simultaneous stabilization problem (SSP)

can be formally stated as given an r-tuple $G_{i}(s), \ldots \ldots G_{r}(s)$

of proper distinct transfer functions for plants, find a compensator C(s) (if it exists) such that all closed-loop

systems $\frac{C(s) G_{i}(s)}{1+C(s) G_{i}(s)},(i=1,2, \ldots \ldots . r)$ are internally

stable $[1,2,3]$.If the compensator $\mathrm{C}(\mathrm{s})$ is further restricted to be stable, i.e. it has no unstable poles, then the simultaneous stabilization problem (SSP) is referred to as a strong simultaneous stabilization problem (SSSP). The requirement for a stable compensator arises in certain cases where the instability of the compensator appears to result in poor overall system sensitivity are to variations in plant parameters as is shown in [4].

The motivation behind the simultaneous control problem is to control a nonlinear system, represented by linear models at different operating points, using one controller. In this case, each linear model represents an operating point. The parameter variations of the system model form a lowfrequency upper bound on the singular values of the loop gain transfer function. . Some of the robust controller design methods, LQG/LTR for example, have no mechanism for dealing with this upper bound. On the other hand, the simultaneous control technique can make gain scheduling easier to implement by reducing the number of operating points to be scheduled. This is accomplished by grouping the total number of pre-specified operating points into classes. A different controller is then designed for each of these classes [5]. Another use of the simultaneous control is in the design of controllers that are robust against sensor or actuator failures. When a sensor or an actuator fails during operation, the system characteristics change, effectively generating a new system. Simultaneous control design can be used to design a single controller that gives good performance for both the original system and the new system generated by the failure.

The simultaneous control problem has attracted many researchers over the last many years. Some of the results for single-input single-output (SISO) transfer functions were reported in [6,7]. Peterson [8] obtained a nonlinear state feedback controller that quadratically stabilized a set of single-input linear systems simultaneously. Schmitendorf [9] acquired a sufficient condition for the existence of a stabilizing linear state feedback for a collection of singleinput linear systems. Miller [10,11] employed linear periodically time-varying controllers for the simultaneous stabilization and disturbance rejection for a set of linear systems. Suryanarayana et al. [12] have also addressed the problem of simultaneously stabilizing controllers for automated vehicles. Strong simultaneous stabilization of linear systems is addressed in [15,16]. Wang et al. [17] and, subsequently, Cai etc al. [18] have also addressed the problem of simultaneous stabilization of nonlinear systems.

In this paper a design technique for the stabilization of $M$ continuous systems by a single output feedback controller has been developed.

Consider the system:

\section{METHODOLOGY}

$\dot{x}=A(q) x+B(q) u$

$y=c(q) x$

Where $q$ is a vector of dimension $\mathrm{v}$, and the value of $q$ determines the system matrices. The above system may represent a linear approximation of a non linear system at different operating points. It may also represent an uncertain plant. In the first case, $q$ is used as an index for identifying operating points while in the second case $q$ may represent intervals of uncertainties of system's parameters. 
Definition 1. The region of operation of the above system is the domain of $q$.

Definition 2. The linear systems

$\dot{x}=A_{i} x+B_{i} u$

$y=c_{\mathrm{i}} x$

$i=1, \ldots M$, represent the system in (1) if the stabilization of the $M$ systems in (2) guarantees the stabilization of (1).

Assume that the $M$ systems in (2) represent the system in (1). We also assume that each of the $M$ systems in (2) is stabilizable by output-feedback controller, and we seek an output-feedback controller $K$ which stabilizes the system in

(1) for some values of $q$. The following theorem shows how to construct such a controller.

Theorem. An output feedback controller $u(t)=K y(t)$ which stabilizes the system in (1) exists if there exist $Q_{i}{ }^{\prime} s$ and $R_{i}{ }^{\prime} s$ such that

$Q_{i}{ }^{\prime}+C_{i}^{t} K_{i}^{t} R_{i} K_{i} C_{i}+C_{i}^{t} \sum_{j \neq i} K_{j}^{t} B_{i}^{t} P_{i}+P_{i} B_{i} \sum_{j \neq i} K_{j} C_{i}>0, i=1, \ldots \ldots, M$

Where $K_{i}$ is given by

$K_{i}=R_{i}^{-1} B_{i}^{t} P_{i} N_{i} C_{i}^{t}\left(C_{i} N_{i} C_{i}^{t}\right)^{-1}$

$P_{i}$ is given by

$\left(A_{i}-B_{i} K_{i} C_{i}\right)^{i} P_{i}+P_{i}\left(A_{i}-B_{i} K_{i} C_{i}\right)+Q_{i}+C_{i}^{t} K_{i}^{t} R_{i} K_{i} C_{i}=0$

And $N_{i}$ is the solution of

$$
N_{i}\left(A_{i}-B_{i} K_{i} C_{i}\right)^{t}+\left(A_{i}-B_{i} K_{i} C_{i}\right) N_{i}+I=0
$$

Proof

Define Lyapunov function $V_{i}=x^{t} P_{i} x$

Where $P_{i}$ is a positive definite matrix. Using the controller in (4), system (1) is stable if the derivatives of $V_{i}$ with respect to time

$$
\dot{V}_{i}=x\left[A_{i}^{t} P_{i}+P_{i} A_{i}\right] x+u B_{i}^{t} P_{i} x+x P_{i} B_{i} u
$$

are negative for all $i=1_{y} \ldots . M$. Choose $P_{i}$ as the solution of (5), the derivative of $V_{i}$ will be negative if and only if (3) is satisfied.

\section{EXAMPLE}

Consider the stabilization of the short-period longitudinal modes of the McDonnell Douglas F4-E aircraft. This is a three-state model. The states are normal acceleration, pitch
ISSN: 2249-6645

rate, and elevator angle. Consider two operating points representing (1) Mach 0.5, altitude 5000ft., and (2) Mach 9, and altitude $35,000 \mathrm{ft}$. The system matrices corresponding to these flight conditions are:

$$
\begin{aligned}
& A_{1}=\left[\begin{array}{ccc}
-.9896 & 17.41 & 96.15 \\
.2648 & -.8512 & -11.39 \\
0.0 & 0.0 & -30.0
\end{array}\right], \\
& A_{2}=\left[\begin{array}{ccc}
-.6607 & 18.11 & 84.34 \\
.08201 & -.6587 & -10.81 \\
0.0 & 0.0 & -30.0
\end{array}\right], \\
& B_{1}=\left[\begin{array}{c}
-97.78 \\
0.0 \\
30.0
\end{array}\right], B_{2}=\left[\begin{array}{c}
-272.2 \\
0.0 \\
30.0
\end{array}\right] \\
& \text { and } \\
& C_{1}=C_{2}=\left[\begin{array}{lll}
1 & 0 & 0 \\
0 & 1 & 0
\end{array}\right]
\end{aligned}
$$

Using the result, the output-feedback controller is

$$
K=\left[\begin{array}{ll}
-4.141 & -2.923
\end{array}\right]_{y}
$$

which is found using (3) - (6). The designed controller stabilizes both operating points. The eigen values of first operating point are $-4.33 .02-1.86 \pm 7.48 j$ and the second operating point has eigenvalues $-135.3,-1.8$ and 20.3. Figs 1 and 2 show the impulse responses of the uncontrolled and controlled system with the designed controller for first as well as for second operating point.

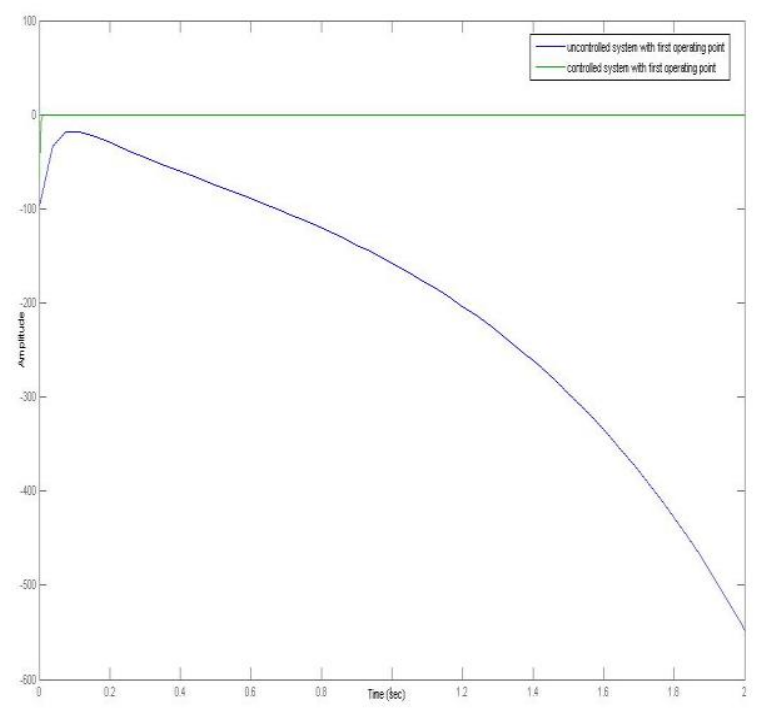

Figure1. Impulse responses of the uncontrolled system and the controlled system with the designed controller at first operating point 


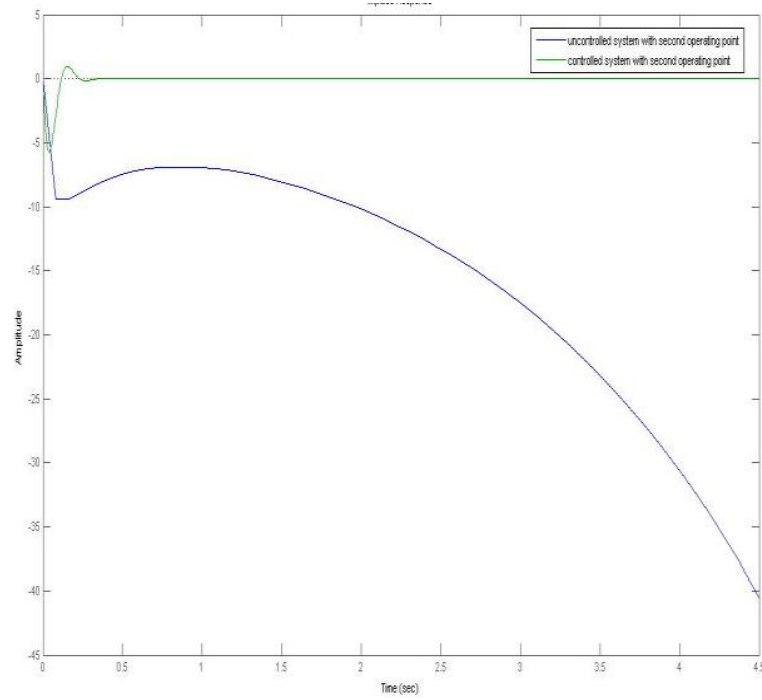

Figure2. Impulse responses of the uncontrolled system and the controlled system with the designed controller at second operating point

\section{CONCLUSION}

The paper has utilized the state and control weighing matrices of the LQR output-feedback controller for solving the problem of stabilization more than one system by a fixed controller. The stabilization of aircraft at two different operating points is presented.

\section{REFERENCES}

[1] V. Blondel , Simultaneous stabilization of Linear Systems (Lecture Notes in Control and Information Sciences 191, Spriger-Verlag, Berlin, 1994).

[2] J.Ackermann, Uncertainty and Control, (Lecture Notes in Control and Information Sciences 70, SpringerVerlag, Berlin, 1985).

[3] Alos, Stabilization of A Class of Plants with Possible Loss of Outputs or Actuator Failures, IEEE Trans. Automatic Control, 28(2), 1983, 231-233.

[4] J. Ackermann, Parameter Space Design of Robust Control Systems, IEEE Trans. Automatic Control, 25(6), 1980, 1058-1082.

[5] Al. F. Sunni, and F. L. Lewis, Gain scheduling simplification by simultaneous stabilization, Trans. ASME: J. Guidance, Control, and Dynamics, 16(3), 1993,602-603.

[6] B.Ghosh and C. Byrns, Simultaneous stabilization and pole placement by nonswitching dynamic compensation, IEEE Trans. Automat. Control, 28, 1983, 735-741.

[7] D.Looze, A dual optimization procedure for linear quadratic robust control problems, Automatica, 19, 1983, 299-302.

[8] I.Peterson, A procedure for simultaneously stabilizing a collection of single input linear systems using nonlinear state-feedback control, Automatica, 23, 1987,33-40.
[9] W. Schimitendorf, and C.Holot, Simultaneous stabilization via state feedback control, IEEE Trans. Automat. Control, 34(9), 1989, 1001-1005.

[10] D. E. Miller, and Chen T.W., Simultaneous stabilization with near-optimal $\mathrm{H}$ performance, IEEE Trans. on Automatic Control, 47(12), 2002, 1986-1998.

[11] D. E. Miller, and M. Rossi, Simultaneous stabilization with near-optimal LQR performance, IEEE Transactions on Automatic Control, 46(10), 2001, 1543-1555.

[12] S. Suryanarayanan ,M. Tomizuka , and T. Suzuki , Design of Simultaneously Stabilizing Controllers and Its Application to Fault-Tolerant Lane -Keeping Controller Design for Automated Vehicles, IEEE Trans. Automatic Control, 12(3), 2004, 329-339.

[13] M.Jing, and M.Kun, Simultaneous stabilization for a class of generalized linear systems, Ninth International Conference on Hybrid Intelligent Systems, 3, 2009, 499503.

[14] S.K. Korovin, A.V. Kudritskii and A.S. Fursor, A remark on the simultaneous $\alpha$-stabilization of linear plants, Differential Equations, 45(5), 2009, 713-720.

[15] Y.Z. Wang, G.Feng, and D.Z. Cheng, Simultaneous stabilization of a set of nonlinear port-controlled Hamiltonian systems, Automatica, 43(3), 2007, 403415.

[16] X.S. Cai, Z.Z.Han and W.Zhang, Simultaneous stabilization for a collection of multi-input nonlinear systems with uncertain parameters, Acta Automatica Sinica, 35(2), 2009, 206-209. 